\title{
Asymptotic Expansion of the Lebesgue Constants Associated With Polynomial Interpolation
}

\author{
By P. N. Shivakumar and R. Wong
}

\begin{abstract}
An infinite asymptotic expansion is obtained for the Lebesgue constants associated with the polynomial interpolation at the zeros of the Chebyshev polynomials. The error due to truncation is shown to be bounded in absolute value by, and of the same sign as, the first neglected term.
\end{abstract}

1. Introduction. Given $n$ distinct points $x_{1 n}, x_{2 n}, \ldots, x_{n n}$ in an interval $[a, b]$ and $n$ real numbers $f\left(x_{1 n}\right), f\left(x_{2 n}\right), \ldots, f\left(x_{n n}\right)$, it is well known that there exists one and only one polynomial $P(x)$ of degree $\leqslant n-1$ such that $P\left(x_{k n}\right)=f\left(x_{k n}\right)$ for $k=$ $1, \ldots, n$; see, e.g., [2] and [9]. Furthermore, this polynomial is explicitly given by the Lagrange interpolation formula

$$
P(x)=\sum_{k=1}^{n} f\left(x_{k n}\right) L_{k}(x),
$$

where the polynomials $L_{k}(x)$ are defined by

$$
L_{k}(x)=\prod_{\substack{i=1 \\ i \neq k}}^{n} \frac{x-x_{i n}}{x_{k n}-x_{i n}}
$$

The Lebesgue constants defined by

$$
\Lambda_{n}=\max _{a \leqslant x \leqslant b} \sum_{k=1}^{n}\left|L_{k}(x)\right|
$$

are closely connected with convergence and divergence properties of the Lagrange interpolation polynomials; see [6], [9] and [10, Section 14.9].

In the case of the special sequence

$$
x_{k n}=\cos (2 k-1) \frac{\pi}{2 n}, \quad k=1, \ldots, n,
$$

$a=-1, b=+1$, i.e., for the zeros of the Chebyshev polynomials, Berstein established in his classical work [1] that

$$
\Lambda_{n} \sim \frac{2}{\pi} \log n, \quad \text { as } n \rightarrow \infty .
$$

Received August 31, 1981.

1980 Mathematics Subject Classification. Primary 41A60; Secondary 41A05.

Key words and phrases. Asymptotic expansion, Lebesgue constant, polynomial interpolation, Chebyshev polynomials. 
This result was later improved by Luttmann and Rivlin [5]. They proved that

$$
\Lambda_{n}=\frac{2}{\pi} \log n+A_{0}+\alpha_{n},
$$

where $\alpha_{n} \rightarrow 0$ as $n \rightarrow \infty$ and

$$
A_{0}=\frac{2}{\pi}\left(\gamma+\log \frac{8}{\pi}\right)
$$

In a recent paper [4], Günttner further improved the result by showing that the error $\alpha_{n}$ in fact satisfies the estimates

$$
0<\alpha_{n}<\frac{\pi}{72 n^{2}}, \quad n \geqslant 1
$$

The purpose of this note is to present a complete asymptotic expansion for $\Lambda_{n}$. More specifically, we demonstrate that, as $n \rightarrow \infty$,

$$
\Lambda_{n} \sim \frac{2}{\pi} \log n+A_{0}+\frac{8}{\pi} \sum_{s=1}^{\infty} \frac{(-1)^{s+1} A_{s}}{(2 n)^{2 s}}
$$

where

$$
A_{s}=\left(2^{2 s-1}-1\right)^{2} \frac{\pi^{2 s}}{2 s} \frac{B_{2 s}^{2}}{(2 s) !}
$$

and the $B_{s}$ 's are the Bernoulli numbers. Furthermore, we prove that the error in stopping the series at any time has the same sign as, and is in absolute value less than, the first term neglected.

2. The Euler-Maclaurin Formula. The well-known Euler-Maclaurin formula states that

$$
\begin{aligned}
\sum_{j=0}^{n} f(j)= & \int_{0}^{n} f(x) d x+\frac{1}{2}[f(0)+f(n)] \\
& +\sum_{s=1}^{m-1} \frac{B_{2 s}}{(2 s) !}\left\{f^{(2 s-1)}(n)-f^{(2 s-1)}(0)\right\}+R_{m}(n),
\end{aligned}
$$

where $m$ and $n$ are arbitrary positive integers,

$$
R_{m}(n)=\int_{0}^{n} \frac{B_{2 m}-B_{2 m}(x-[x])}{(2 m) !} f^{(2 m)}(x) d x,
$$

and $B_{2 m}(x)$ is the Bernoulli polynomial. Furthermore, if $f^{(2 m)}(x)$ and $f^{(2 m+2)}(x)$ have the same constant sign in $(0, n)$, then $R_{m}(n)$ is bounded in absolute value by, and has the same sign as, the first neglected term in (2.1); see [7, p. 285].

Now put

$$
P_{2 m}(x)=\frac{1}{(2 m) !} B_{2 m}(x-[x])
$$

and note that $P_{2 m}(x)$ is a periodic function with period 1. The following observations were made by Günttner [4]:

$$
\begin{gathered}
P_{2 m}(2 x)=2^{2 m-1}\left[P_{2 m}(x)+P_{2 m}\left(x+\frac{1}{2}\right)\right], \\
(-1)^{m+1}\left[P_{2 m}(x)-P_{2 m}\left(\frac{1}{2}\right)\right] \geqslant 0
\end{gathered}
$$




$$
\begin{gathered}
\sum_{j=1}^{n} f(2 j-1)=\sum_{j=0}^{2 n} f(j)-\sum_{j=0}^{n} f(2 j), \\
\frac{1}{2} \int_{0}^{2 n} f(x) d x=\int_{0}^{2 n} f(x) d x-\int_{0}^{n} f(2 x) d x .
\end{gathered}
$$

Equation (2.4) is a special case of the multiplication theorem [7, p. 285, Ex. 1.4], and (2.5) follows from the fact that $(-1)^{m+1} P_{2 m}(x)$ assumes its absolute minimum in $[0,1]$ at $x=\frac{1}{2}$; see the properties of the Bernoulli polynomials in [7, pp. 281-283]. A combination of the above identities gives

$$
\begin{aligned}
\sum_{j=1}^{n} f(2 j-1)= & \frac{1}{2} \int_{0}^{2 n} f(x) d x+\sum_{s=1}^{m-1} \frac{B_{2 s}}{(2 s) !}\left(1-2^{2 s-1}\right) \\
& \times\left[f^{(2 s-1)}(2 n)-f^{(2 s-1)}(0)\right]+r_{m}(n),
\end{aligned}
$$

where

$$
r_{m}(n)=-2^{2 m} \int_{0}^{n} f^{(2 m)}(2 x)\left[P_{2 m}\left(x+\frac{1}{2}\right)-P_{2 m}\left(\frac{1}{2}\right)\right] d x .
$$

From (2.5) it also follows that if $f^{(2 m)}(x)$ and $f^{(2 m+2)}(x)$ have the same constant sign in $(0,2 n)$, then $r_{m}(n)$ is bounded in absolute value by, and has the same sign as, the first neglected term in (2.8); cf. the so-called error test in asymptotics [7, p. 68].

Example 1. Let $k$ be a positive integer and $f(x)=x^{k}$. From (2.8) (and the remark following it), it follows that, for any $m=1, \ldots,\left[\frac{1}{2} k\right]+1$, we have

$$
\sum_{j=0}^{n-1}(2 j+1)^{k}=\frac{(2 n)^{k+1}}{k+1} \sum_{s=0}^{m-1}\left(\begin{array}{c}
k+1 \\
2 s
\end{array}\right)\left(1-2^{2 s-1}\right) \frac{B_{2 s}}{(2 n)^{2 s}}+\varepsilon_{m}^{(k)}(n),
$$

where $\varepsilon_{m}^{(k)}(n)=0$ if $m=\left[\frac{1}{2} k\right]+1$ and

$$
0 \leqslant(-1)^{m} \varepsilon_{n}^{(k)}(n) \leqslant \frac{(2 n)^{k+1}}{k+1}\left(\begin{array}{c}
k+1 \\
2 m
\end{array}\right)\left(2^{2 m-1}-1\right) \frac{\left|B_{2 m}\right|}{(2 n)^{2 m}}
$$

if $m<\left[\frac{1}{2} k\right]+1$.

Example 2. It is well known that, as $n \rightarrow \infty$,

$$
\sum_{j=1}^{n-1} \frac{1}{j} \sim \log n+\gamma-\frac{1}{2 n}-\sum_{s=1}^{\infty} \frac{B_{2 s}}{2 s} \frac{1}{n^{2 s}},
$$

where $\gamma$ denotes Euler's constant. Furthermore, if the expansion is truncated at the term $s=m-1$, where $m$ is a positive integer, then the remainder is bounded in absolute value by the next term and has the same sign; see [7, p. 292]. From this and (2.6), it follows that

$$
\sum_{j=0}^{n-1} \frac{1}{2 j+1} \sim \frac{1}{2} \log n+\log 2+\frac{\gamma}{2}-\sum_{s=1}^{\infty} \frac{B_{2 s}}{2 s} \frac{\left(1-2^{2 s-1}\right)}{(2 n)^{2 s}},
$$

as $n \rightarrow \infty$. However, the error analysis for the expansion (2.12) no longer applies to the expansion (2.13). (All one can conclude at the moment is that the remainder is bounded in absolute value by three times the first neglected term.) To obtain a 
similar result for (2.13), we employ (2.8)-(2.9) with $f(x)=1 /(x+2)$, which together with (2.13) gives

$$
\sum_{j=0}^{n-1} \frac{1}{2 j+1}=\frac{1}{2} \log n+\log 2+\frac{\gamma}{2}-\sum_{s=1}^{m-1} \frac{B_{2 s}}{2 s} \frac{\left(1-2^{2 s-1}\right)}{(2 n)^{2 s}}+\delta_{m}(n)
$$

with

$$
\delta_{m}(n)=2^{2 m}(2 m) ! \int_{n-1}^{\infty} \frac{P_{2 m}\left(x+\frac{1}{2}\right)-P_{2 m}\left(\frac{1}{2}\right)}{(2 x+2)^{2 m+1}} d x .
$$

In view of (2.5) and the error test [7, p. 68], we have

$$
0 \leqslant(-1)^{m+1} \delta_{m}(n) \leqslant \frac{\left|B_{2 m}\right|}{2 m} \frac{\left(2^{2 m-1}-1\right)}{(2 n)^{2 m}} .
$$

Example 3. Let $\alpha$ be any real number, not -1 or a positive integer. An argument completely analogous to that in Example 2 yields

$$
\begin{aligned}
& \sum_{j=0}^{n-1}(2 j+1)^{\alpha}-\left(1-2^{\alpha}\right) \zeta(-\alpha) \\
& \sim \frac{(2 n)^{\alpha+1}}{\alpha+1} \sum_{s=0}^{\infty}\left(\begin{array}{c}
\alpha+1 \\
2 s
\end{array}\right)\left(1-2^{2 s-1}\right) \frac{B_{2 s}}{(2 n)^{2 s}},
\end{aligned}
$$

as $n \rightarrow \infty$, where $\zeta(z)$ is the Riemann Zeta function. Furthermore, the remark in Example 2, concerning the error due to truncation, also holds here if $m>\frac{1}{2}(\alpha+1)$.

3. Proof of (1.4). In [3], Ehlich and Zeller proved the identity

$$
\Lambda_{n}=\frac{1}{n} \sum_{j=0}^{n-1} \cot \frac{(2 j+1) \pi}{4 n}
$$

see also [8]. Following Günttner, we substitute the expansion

$$
\cot z=\frac{1}{z}-\sum_{r=1}^{\infty} 2^{2 r}\left|B_{2 r}\right| \frac{z^{2 r-1}}{(2 r) !} \quad(|z|<\pi)
$$

in (3.1). Thus

$$
\Lambda_{n}=\frac{4}{\pi} \sum_{j=0}^{n-1} \frac{1}{2 j+1}-\frac{4}{\pi} \sum_{r=1}^{\infty} \frac{\left|B_{2 r}\right|}{(2 r) !} \frac{\pi^{2 r}}{(2 n)^{2 r}} \sum_{j=0}^{n-1}(2 j+1)^{2 r-1} .
$$

From (2.10) and (2.14) it now follows that

$$
\Lambda_{n}=\frac{2}{\pi} \log n+A_{0}+\frac{8}{\pi} \sum_{s=1}^{m-1} \frac{(-1)^{s+1} A_{s}}{(2 n)^{2 s}}+\rho_{m}(n),
$$

where

$$
A_{0}=\frac{4}{\pi} \log 2+\frac{2}{\pi} \gamma-\frac{1}{\pi} \sum_{r=1}^{\infty} \frac{\pi^{2 r}\left|B_{2 r}\right|}{r \cdot(2 r) !}
$$




$$
\begin{aligned}
(-1)^{s+1} A_{s}= & \frac{B_{2 s}}{2 s} \frac{\left(2^{2 s-1}-1\right)}{2} \\
& +\frac{B_{2 s}}{(2 s) !} \frac{2^{2 s-1}-1}{2} \sum_{r=s+1}^{\infty} \frac{\left|B_{2 r}\right|}{(2 r-2 s) !} \frac{\pi^{2 r}}{2 r}
\end{aligned}
$$

and

$$
\rho_{m}(n)=\frac{4}{\pi}\left[\delta_{m}(n)-\sum_{r=m+1}^{\infty} \frac{\left|B_{2 r}\right|}{(2 r) !}\left(\frac{\pi}{2 n}\right)^{2 r} \varepsilon_{m}^{(2 r-1)}(n)\right] .
$$

To show that (3.5) and (3.6) agree with (1.2) and (1.5), respectively, we recall the identities

$$
\begin{aligned}
& \log \sin z=\log z-\sum_{r=1}^{\infty} \frac{2^{2 r-1}\left|B_{2 r}\right|}{r \cdot(2 r) !} z^{2 r} \quad(0<|z|<\pi), \\
& \tan z=\sum_{r=1}^{\infty} \frac{2^{2 r}\left(2^{2 r}-1\right)}{(2 r) !}\left|B_{2 r}\right| z^{2 r-1} \quad\left(|z|<\frac{\pi}{2}\right) .
\end{aligned}
$$

((3.8) is obtained from (3.2) by integration.) Replacing $z$ by $\pi / 2$ in (3.8), we obtain immediately (1.2) from (3.5). Since $\cot (\pi / 2+z)=-\tan z$, it follows from (3.9) that

$$
\cot ^{(2 r-1)}\left(\frac{\pi}{2}\right)=-2^{2 r}\left(2^{2 r}-1\right)\left|B_{2 r}\right| / 2 r .
$$

On the other hand, we have from (3.2)

$$
\cot ^{(2 r-1)}\left(\frac{\pi}{2}\right)=-(2 r-1) !\left(\frac{2}{\pi}\right)^{2 r}-\left(\frac{2}{\pi}\right)^{2 r} \sum_{s=r}^{\infty} \frac{\pi^{2 s}}{(2 s-2 r) !} \frac{\left|B_{2 s}\right|}{2 s}
$$

A comparison of (3.10) and (3.11) gives

$$
\sum_{s=r+1}^{\infty} \frac{\left|B_{2 s}\right|}{2 s} \frac{\pi^{2 s}}{(2 s-2 r) !}=\frac{\pi^{2 r}}{r}\left(2^{2 r-1}-1\right)\left|B_{2 r}\right|-(2 r-1) !,
$$

which implies that (3.6) and (1.5) agree. To estimate the remainder $\rho_{m}(n)$, we apply (2.11) and (2.16) to (3.7). The result is

$$
0 \leqslant(-1)^{m+1} \rho_{m}(n) \leqslant \frac{8}{\pi} \frac{\left(2^{2 m}-1\right)^{2}}{(2 m) !} \frac{\pi^{2 m}}{2 m} \frac{B_{2 m}^{2}}{(2 n)^{2 m}} .
$$

Here we have also made use of (3.12). This completes the proof of the result stated in Section 1.

Remark. The methods employed in this note are applicable to a much broader class of problems than merely the sum (3.1). It is a trivial extension to apply the method to any Riemann sums of the forms

$$
S_{n}=\frac{1}{n} \sum_{j=0}^{n-1} f\left(\frac{2 j+1}{2 n}\right)
$$

and

$$
S_{n}=\frac{1}{n} \sum_{j=1}^{n-1} f\left(\frac{j}{n}\right)
$$


as long as $f(z)$ has a Laurent expansion of the form

$$
f(z)=\sum_{r=0}^{\infty} c_{r} z^{r-1}, \quad 0<|z|<\rho,
$$

$\rho$ being greater than one.

Department of Applied Mathematics

University of Manitoba

Winnipeg, Manitoba, Canada R3T 2N2

Department of Mathematics

University of Manitoba

Winnipeg, Manitoba, Canada R3T 2N2

1. S. N. BerSTEIN, "Sur la limitation des valeurs d'un polynôme $P(x)$ de degré $n$ sur tout un segment par ses valeurs en $(n+1)$ points de segment," Izv. Akad. Nauk SSSR, v. 7, 1931, pp. 1025-1050.

2. P. J. DAvis, Interpolation and Approximation, Blaisdell, New York, 1965.

3. H. Ehlich \& K. Zeller, "Auswertung der Normen von Interpolationsoperatoren," Math. Ann., v. 164, 1966, pp. 105-112.

4. R. GüNTTNER, "Evaluation of Lebesgue constants," SIAM J. Numer. Anal., v. 17, 1980, pp. $512-520$.

5. F. W. LutTmann \& T. J. Rivis, "Some numerical experiments in the theory of polynomial interpolation," IBM J. Res. Develop., v. 9, 1965, pp. 187-191.

6. I. P. Natanson, Constructive Function Theory, Vol. III, Ungar, New York, 1965.

7. F. W. J. Olver, Asymptotics and Special Functions, Academic Press, New York, 1974.

8. M. J. D. Powel, " On the maximum errors of polynomial approximations defined by interpolation and by least square criteria," Comput. J., v. 9, 1967, pp. 404-407.

9. T. J. Rivuin, The Chebyshev Polynomials, Wiley-Interscience, New York, 1974.

10. G. Szegö, Orthogonal Polynomials, 4th ed., Amer. Math. Soc. Colloq. Publ., vol. 23, Amer. Math. Soc., Providence, R.I., 1975. 